

Л. Л. Оридорога, С. Хасси, Теоремы полноты для операторов типа Дирака с граничными условиями общего вида, зависящими от спектрального параметра, Матем. заметки, 2003, том 74, выпуск 2, 316-320

DOI: https://doi.org/10.4213/mzm593

Использование Общероссийского математического портала Math-Net.Ru подразумевает, что вы прочитали и согласны с пользовательским соглашением http://www.mathnet.ru/rus/agreement

Параметры загрузки:

IP : 54.198 .187 .58

26 апреля 2023 г., 03:13:27

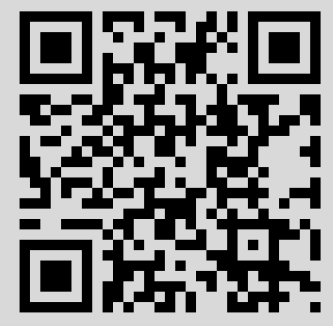




\section{ТЕОРЕМЫ ПОЛНОТЫ ДЛЯ ОПЕРАТОРОВ ТИПА ДИРАКА С ГРАНИЧНЫМИ УСЛОВИЯМИ ОБЩЕГО ВИДА, ЗАВИСЯЩИМИ ОТ СПЕКТРАЛЬНОГО ПАРАМЕТРА}

\section{Л. Л. Оридорога, С. Хасси}

1. Введение. Хорошо известно (см. [1]), что система собственных и присоединенных функций $(\mathrm{CСП \Phi )} \mathrm{задачи} \mathrm{Штурма-Лиувилля}$

$$
-y^{\prime \prime}+q(x) y=\lambda^{2} y
$$

с разделяюшимися краевыми условиями

$$
y^{\prime}(0)-h_{0} y(0)=y^{\prime}(1)-h_{1} y(1)=0
$$

полна в пространстве $L_{2}[0,1]$ при произвольном комплекснозначном потенциале $q \in L_{1}[0,1]$ и любых $h_{0}, h_{1} \in \mathbb{C}$. Там же показана полнота ССПФ в случае любых (а не только разделяющихся) невырожденных граничных условий.

Полнота ССП $\Phi$ дифференциального уравнения

$$
y^{(n)}+\sum_{j=0}^{n-2} q_{j}(x) y=\lambda^{n} y
$$

произвольного порядка с разделяющимися (нерегулярными) краевыми условиями была анонсирована Келдышем в известной работе [2] и впервые доказана Шкаликовым в [3].

В [4] этот результат [1] был обобщен на системы дифференциальных уравнений первого порядка с граничными условиями общего вида (не зависящими от спектрального параметра).

В статьях [5] и [6] исследована полнота ССПФ уравнения (1) для случая нелинейных граничных условий, зависящих от спектрального параметра следующим образом:

$$
\left\{\begin{array}{l}
P_{11}(\lambda) y(0)+P_{12}(\lambda) y^{\prime}(0)=0 \\
P_{21}(\lambda) y^{2}\left(\frac{1}{2}\right)+P_{22}(\lambda) y\left(\frac{1}{2}\right) y^{\prime}\left(\frac{1}{2}\right)+P_{23}(\lambda) y^{\prime 2}\left(\frac{1}{2}\right)=0
\end{array}\right.
$$

и

$$
\begin{aligned}
& P_{i 0}(\lambda) y^{2}(0)+P_{i 1}(\lambda) y^{\prime 2}(0)+P_{i 2}(\lambda) y^{2}\left(\frac{1}{3}\right)+P_{i 3}(\lambda) y^{\prime 2}\left(\frac{1}{3}\right)+P_{i 4}(\lambda) y(0) y^{\prime}(0) \\
& \quad+P_{i 5}(\lambda) y(0) y\left(\frac{1}{3}\right)+P_{i 6}(\lambda) y(0) y^{\prime}\left(\frac{1}{3}\right)+P_{i 7}(\lambda) y^{\prime}(0) y\left(\frac{1}{3}\right) \\
& \quad+P_{i 8}(\lambda) y^{\prime}(0) y^{\prime}\left(\frac{1}{3}\right)+P_{i 9}(\lambda) y\left(\frac{1}{3}\right) y^{\prime}\left(\frac{1}{3}\right)=0, \quad i=1,2 .
\end{aligned}
$$

В статьях [7] и [8] был получен аналогичный результат для систем типа Дирака с разделяюшимися краевыми условиями (как линейньми, так и нелинейными, аналогичными условиям (4)), зависящими от спектрального параметра.

В данной работе приводятся (с краткими доказательствами) теоремы полноты ССПФ для систем двух интегродифференциальных уравнений первого порядка с линейными или квадратичными граничными условиями общего вида, зависящими от спектрального параметра. Именно, пусть

Работа первого автора поддержана Финской Академией, проект 52528. 
$a<0<b$ и $B=\operatorname{diag}\left(a^{-1}, b^{-1}\right)$ - диагональная матрица размера $2 \times 2$. Рассмотрим в пространстве $L_{2}[0,1] \oplus L_{2}[0,1]$ граничную задачу для системы интегродифференциальных уравнений вида

$$
\frac{1}{i} B y^{\prime}+Q(x) y+\int_{0}^{x} M(x, t) y(t) d t=\lambda y,
$$

где

$$
Q(x, t)=\left(\begin{array}{cc}
0 & q_{1} \\
q_{2} & 0
\end{array}\right), \quad M(x, t)=\left(\begin{array}{ll}
M_{11} & M_{12} \\
M_{21} & M_{22}
\end{array}\right), \quad y(x)=\left(\begin{array}{l}
y_{1}(x) \\
y_{2}(x)
\end{array}\right)
$$

При этом предполагается, что $q_{j} \in L_{1}[0,1]$ и $M_{i j} \in L_{\infty}(\Omega)$, где $\Omega=\{0 \leqslant t \leqslant x \leqslant 1\}, i, j=1,2$.

Рассматриваются следующие типы граничных условий, зависяших от спектрального параметpa:

(i) линейные условия общего вида

$$
P_{i 1}(\lambda) y_{1}(0)+P_{i 2}(\lambda) y_{2}(0)+P_{i 3}(\lambda) y_{1}(1)+P_{i 4}(\lambda) y_{2}(1)=0, \quad i=1,2 ;
$$

(ii) квадратичные условия общего вида

$$
\begin{aligned}
& P_{i 0}(\lambda) y_{1}^{2}(0)+P_{i 1}(\lambda) y_{2}^{2}(0)+P_{i 2}(\lambda) y_{1}^{2}\left(\frac{1}{2}\right)+P_{i 3}(\lambda) y_{2}^{2}\left(\frac{1}{2}\right)+P_{i 4}(\lambda) y_{1}(0) y_{2}(0) \\
& \quad+P_{i 5}(\lambda) y_{1}(0) y_{1}\left(\frac{1}{2}\right)+P_{i 6}(\lambda) y_{1}(0) y_{2}\left(\frac{1}{2}\right)+P_{i 7}(\lambda) y_{2}(0) y_{1}\left(\frac{1}{2}\right) \\
& \quad+P_{i 8}(\lambda) y_{2}(0) y_{2}\left(\frac{1}{2}\right)+P_{i 9}(\lambda) y_{1}\left(\frac{1}{2}\right) y_{2}\left(\frac{1}{2}\right)=0, \quad i=1,2
\end{aligned}
$$

где $P_{i j}(\lambda)$ - некоторые многочлены.

В следующей работе будут приведены результаты о полноте ССПФ задач (6), (7) и (6), (8) для систем типа Дирака произвольного порядка $n$.

2. Вспомогательные результаты. Для доказательства основных теорем данной работы используется следующая лемма, дающая оценки скорости роста функций

$$
\vec{\varphi}_{0}(x ; \lambda)=\left(\begin{array}{l}
\varphi_{01}(x ; \lambda) \\
\varphi_{02}(x ; \lambda)
\end{array}\right) \quad \text { и } \quad \vec{\psi}_{0}(x ; \lambda)=\left(\begin{array}{c}
\psi_{01}(x ; \lambda) \\
\psi_{02}(x ; \lambda)
\end{array}\right)
$$

являющихся решениями задачи Коши для системы (6) с начальными условиями

$$
\varphi_{01}(0 ; \lambda)=\psi_{02}(0 ; \lambda)=1 \quad \text { и } \quad \varphi_{02}(0 ; \lambda)=\psi_{01}(0 ; \lambda)=0
$$

соответственно.

Лемма 1. Функиии $\vec{\varphi}_{0}(x ; \lambda) u \vec{\psi}_{0}(x ; \lambda)$ удовлетворяют оценкам

$$
\begin{array}{ll}
\varphi_{01}(x ; \lambda)=\left(1+O\left(\frac{1}{\operatorname{Im} \lambda}\right)\right) \exp (a \lambda i x), & \varphi_{02}(x ; \lambda)=O\left(\frac{1}{\operatorname{Im} \lambda}\right) \exp (a \lambda i x), \\
\psi_{01}(x ; \lambda)=O\left(\frac{1}{\operatorname{Im} \lambda}\right) \exp (a \lambda i x), & \psi_{02}(x ; \lambda)=O\left(\frac{1}{\operatorname{Im} \lambda}\right) \exp (a \lambda i x)
\end{array}
$$

при $\lambda \in \mathbb{C}^{+}$, и удовлетворяют оченкам

$$
\begin{array}{ll}
\varphi_{01}(x ; \lambda)=O\left(\frac{1}{\operatorname{Im} \lambda}\right) \exp (b \lambda i x), & \varphi_{02}(x ; \lambda)=O\left(\frac{1}{\operatorname{Im} \lambda}\right) \exp (b \lambda i x), \\
\psi_{01}(x ; \lambda)=O\left(\frac{1}{\operatorname{Im} \lambda}\right) \exp (b \lambda i x), & \psi_{02}(x ; \lambda)=\left(1+O\left(\frac{1}{\operatorname{Im} \lambda}\right)\right) \exp (b \lambda i x)
\end{array}
$$

nрu $\lambda \in \mathbb{C}^{-}$.

В доказательстве леммы использются построенные в [9] треугольные операторы преобразования, переводящие решения задачи Коши для простейшей системы типа (6), но с нулевой потенциальной матрицей $Q$, в решения задачи Коши для системы (6) с теми же начальными данньми. 


\section{3. Теоремы полноты.}

Tеорема 2. Пусть $P_{i j}, i=1,2, j=1,2,3,4$, - многочлень такие, что при всех $\lambda \in \mathbb{C}$ ранг матрицы

$$
P(\lambda)=\left(\begin{array}{llll}
P_{11}(\lambda) & P_{12}(\lambda) & P_{13}(\lambda) & P_{14}(\lambda) \\
P_{21}(\lambda) & P_{22}(\lambda) & P_{23}(\lambda) & P_{24}(\lambda)
\end{array}\right)
$$

равен 2 и, кроме того,

$$
\operatorname{deg} J_{14}=\operatorname{deg} J_{32} \geqslant \max \left\{\operatorname{deg} J_{13}, \operatorname{deg} J_{42}, M\right\},
$$

əде $M=\max \left\{\operatorname{deg} P_{i j}: i \in\{1,2\}, j \in\{1,2,3,4\}\right\} u$

$$
J_{i j}=\operatorname{det}\left(\begin{array}{ll}
P_{1 i} & P_{1 j} \\
P_{2 i} & P_{2 j}
\end{array}\right), \quad i, j \in\{1,2,3,4\}
$$

Тогда ССПФ задачи (6), (7) полна в пространстве $L^{2}[0,1] \oplus L^{2}[0,1]$.

Более того, пусть мнохество $\Phi$, состоящее из $N:=\operatorname{deg} J_{14}-M$ собственных и присоединенных функиий, удовлетворяет следующему условию: если Ф содержит некоторую собственную или присоединенную функиию, соответствующ,ую собственному значению $\lambda_{k}$, то вместе с ней оно содержит и все присоединенные функиии более высокого порядка, соответствующ,ие тому же собственному значению. Тогда ССПФ задачи (6), (7) без множества $\Phi$ такжсе полна в пространстве $L_{2}[0,1] \oplus L_{2}[0,1]$.

ДокАЗАТЕЛЬСтво. Пусть $\chi(\lambda)$ - характеристическая функция задачи $(6),(7)$ :

$$
\chi(\lambda):=\operatorname{det}\left(\begin{array}{ll}
Q_{11}(\lambda) & Q_{12}(\lambda) \\
Q_{21}(\lambda) & Q_{22}(\lambda)
\end{array}\right)
$$

где

$$
\begin{aligned}
& Q_{11}(\lambda)=P_{11}(\lambda)+P_{13}(\lambda) \varphi_{01}(1 ; \lambda)+P_{14}(\lambda) \varphi_{02}(1 ; \lambda), \\
& Q_{12}(\lambda)=P_{12}(\lambda)+P_{13}(\lambda) \psi_{01}(1 ; \lambda)+P_{14}(\lambda) \psi_{02}(1 ; \lambda), \\
& Q_{21}(\lambda)=P_{21}(\lambda)+P_{23}(\lambda) \varphi_{01}(1 ; \lambda)+P_{24}(\lambda) \varphi_{02}(1 ; \lambda), \\
& Q_{22}(\lambda)=P_{22}(\lambda)+P_{23}(\lambda) \psi_{01}(1 ; \lambda)+P_{24}(\lambda) \psi_{02}(1 ; \lambda) .
\end{aligned}
$$

Число $\lambda$ является собственным для оператора (6), (7) тогда и только тогда, когда $\chi(\lambda)=0$. И, более того, если $\lambda_{0}$ - корень $\chi(\lambda)$ порядка $p$, то оператор (6), (7) имеет ровно $p$ собственных и присоединенных функций, соответствующих собственному значению $\lambda_{0}$.

Предположим, что существует ненулевая вектор-функция $\vec{f}(x)=\left(f_{1}(x), f_{2}(x)\right)^{\top}$, ортогональная ССПФ задачи (6), (7) (кроме, быть может, функций из множества $\Phi$ ). Рассмотрим функцию

$$
\widetilde{F_{j}}(\lambda):=\left\langle\vec{\omega}_{j}(x ; \lambda), \vec{f}(x)\right\rangle=\int_{0}^{1}\left(\omega_{j 1}(x ; \lambda) \overline{f_{1}(x)}+\omega_{j 2}(x ; \lambda) \overline{f_{2}(x)}\right) d x
$$

где

$$
\begin{aligned}
\vec{\omega}_{j}(x ; \lambda)= & \left(P_{j 2}(\lambda)+P_{j 3}(\lambda) \psi_{01}(1 ; \lambda)+P_{j 4}(\lambda) \psi_{02}(1 ; \lambda)\right) \vec{\varphi}_{0}(x ; \lambda) \\
& -\left(P_{j 1}(\lambda)+P_{j 3}(\lambda) \varphi_{01}(1 ; \lambda)+P_{j 4}(\lambda) \varphi_{02}(1 ; \lambda)\right) \vec{\psi}_{0}(x ; \lambda), \quad j=1,2 .
\end{aligned}
$$

Очевидно, что $\widetilde{F_{j}}(\lambda)$ - целая функция. Если $\lambda_{s}$ - собственное значение оператора $(6),(7)$ порядка $p$ и множество $\Phi$ содержит $k$ (возможно, $k=0$ ) собственных и присоединенных функций, соответствующих собственному значению $\lambda_{0}$, то $\lambda_{s}$ является корнем функций $\widetilde{F_{j}}(\lambda), j=1,2$, порядка не ниже $p-k$.

Пусть $\Lambda$ - множество таких собственных чисел задачи (6), (7), что соответствующие им собственные или присоединенные функции входят в множество $\Phi$. Для каждого $\lambda_{s} \in \Lambda$ обозначим 
через $p_{s}$ количество собственных и присоединенных функций, соответствующих $\lambda_{s}$, которые содержатся в множестве $\Phi$. Пусть

$$
\Pi(\lambda)=\prod_{\lambda_{s} \in \Lambda}\left(\lambda-\lambda_{s}\right)^{p_{s}}
$$

Пусть $\lambda_{k}$ - собственное значение оператора (6), (7) кратности $p$. Тогда $\lambda_{k}$ является корнем кратности не ниже $p$ произведения $\Pi(\lambda) \widetilde{F}_{j}(\lambda)$. Следовательно, функция

$$
F_{j}(\lambda)=\frac{\Pi(\lambda) \widetilde{F_{j}}(\lambda)}{\chi(\lambda)}
$$

целая.

Оценим ее. Перепишем для этого $\chi(\lambda)$ следующим образом:

$$
\begin{aligned}
\chi(\lambda)= & J_{12}+J_{13} \psi_{01}(1 ; \lambda)+J_{14} \psi_{02}(1 ; \lambda)+J_{32} \varphi_{01}(1 ; \lambda)+J_{42} \varphi_{02}(1 ; \lambda) \\
& +J_{34} \operatorname{det}\left(\begin{array}{cc}
\varphi_{01}(1 ; \lambda) & \psi_{01}(1 ; \lambda) \\
\varphi_{02}(1 ; \lambda) & \psi_{02}(1 ; \lambda)
\end{array}\right) \\
= & J_{12}+J_{13} \psi_{01}(1 ; \lambda)+J_{14} \psi_{02}(1 ; \lambda)+J_{32} \varphi_{01}(1 ; \lambda)+J_{42} \varphi_{02}(1 ; \lambda) \\
& +J_{34}\left(1+O\left(\frac{1}{\operatorname{Im} \lambda}\right)\right) \exp ((a+b) \lambda i) .
\end{aligned}
$$

Из оценок $(11),(12)$ и условия (14) получаем следующие оценки $\chi(\lambda)$ :

$$
\begin{array}{ll}
\chi(\lambda)=\left(1+O\left(\frac{1}{\operatorname{Im} \lambda}\right)\right) J_{32} \exp (a \lambda i), & \lambda \in \mathbb{C}^{+}, \\
\chi(\lambda)=\left(1+O\left(\frac{1}{\operatorname{Im} \lambda}\right)\right) J_{14} \exp (b \lambda i), & \lambda \in \mathbb{C}^{-} .
\end{array}
$$

Из определения функций $\vec{\omega}_{j}(x ; \lambda)$ получаем

$$
\begin{aligned}
\vec{\omega}_{j}(x ; \lambda)= & -P_{j 1}(\lambda) \psi_{0}(x ; \lambda)+P_{j 2}(\lambda) \varphi_{0}(x ; \lambda) \\
& +\left(1+O\left(\frac{1}{\operatorname{Im} \lambda}\right)\right) \exp ((a+b) \lambda i)\left(-P_{j 3}(\lambda) \psi_{1}(x ; \lambda)+P_{j 4}(\lambda) \varphi_{1}(x ; \lambda)\right) .
\end{aligned}
$$

При $\lambda \in \mathbb{C}^{+}$из $(25)$ и оценок (11) получаем

$$
\begin{aligned}
\omega_{j k}(x ; \lambda)= & \left(O\left(P_{j 1}(\lambda)\right)+O\left(P_{j 2}(\lambda)\right)\right) \exp (a \lambda i x) \\
& +\left(O\left(P_{j 3}(\lambda)\right)+O\left(P_{j 4}(\lambda)\right)\right) \exp (a \lambda i) \exp (b \lambda i x) .
\end{aligned}
$$

Поскольку при таких $\lambda$

$$
\begin{aligned}
& \int_{0}^{1}\left|f_{k}(x) \exp (a \lambda i x)\right| d x=O\left(\exp (a \lambda i)|\operatorname{Im} \lambda|^{-1 / 2}\right) \text { и } \\
& \int_{0}^{1}\left|f_{k}(x) \exp (b \lambda i x)\right| d x=O\left(|\operatorname{Im} \lambda|^{-1 / 2}\right),
\end{aligned}
$$

то существует константа $c_{1}>0$ такая, что при $\operatorname{Im} \lambda>c_{1}$

$$
\widetilde{F}_{j}(\lambda)=O\left(\max \left|P_{j k}(\lambda)\right| \exp (a \lambda i)|\operatorname{Im} \lambda|^{-1 / 2}\right)=O\left(\lambda^{M} \exp (a \lambda i)|\operatorname{Im} \lambda|^{-1 / 2}\right) .
$$

Аналогично, существует константа $c_{2}<0$ такая, что при $\operatorname{Im} \lambda<c_{2}$

$$
\widetilde{F}_{j}(\lambda)=O\left(\lambda^{M} \exp (b \lambda i)|\operatorname{Im} \lambda|^{-1 / 2}\right) .
$$

Окончательно, из $(23),(24),(27)$ и (28) получаем следующую оценку:

$$
F_{j}(\lambda)=O\left(|\operatorname{Im} \lambda|^{-1 / 2}\right), \quad|\operatorname{Im} \lambda|>c .
$$

Поэтому, по теореме Фрагмена-Линделёфа для полосы, $F_{i}(\lambda) \equiv 0$. Следовательно, и $\widetilde{F}_{i}(\lambda) \equiv 0$, т.е. $\vec{f}(x)$ ортогональна обеим функциям $\vec{\omega}_{j}(x ; \lambda)$ при всех $\lambda$, а значит и всем решениям уравнения (6). Поэтому $\vec{f}(x) \equiv 0$, т.е. ССПФ задачи (6), (7) без множества $\Phi$ полна в пространстве $L^{2}[0,1] \oplus L^{2}[0,1]$. 
Теорема 3. Пусть $P_{i j}, i=1,2, j=0,1, \ldots, 9,-$ многочлены такие, что

$$
\operatorname{rank}\left(\begin{array}{llll}
P_{10}(\lambda) & P_{11}(\lambda) & \ldots & P_{19}(\lambda) \\
P_{20}(\lambda) & P_{21}(\lambda) & \ldots & P_{29}(\lambda)
\end{array}\right)=2, \quad \lambda \in \mathbb{C}
$$

u, кроме того,

$$
\operatorname{deg} J_{03}=\operatorname{deg} J_{12}=M,
$$

əəe

$$
J_{i j}=\operatorname{det}\left(\begin{array}{ll}
P_{1 i} & P_{1 j} \\
P_{2 i} & P_{2 j}
\end{array}\right), \quad i, j=0,1, \ldots, 9
$$

u $M=\max \left\{\operatorname{deg} P_{i j}: i \in\{1,2\} ; j \in\{0,1, \ldots, 9\}\right\}$. Тогда ССПФ задачи (6), (8) полна в пространстве $L^{2}[0,1] \oplus L^{2}[0,1]$.

Более того, пусть мнохсество $\Phi$, состоящее из $M$ собственных и присоединенных функиий, удовлетворяет следующему условию: если Ф содержит некоторую собственную или присоединенную функиию, соответствуюшую собственному значению $\lambda_{k}$, то вместе с ней оно содержит и все присоединенные функиии более высокого порядка, соответствуюшие тому же собственному значению. Тогда ССПФ задачи (6), (8) без множества $\Phi$ также полна в пространстве $L_{2}[0,1] \oplus L_{2}[0,1]$.

ДоКАЗАТЕЛЬСтво этой теоремы аналогично доказательству теоремы 2.

\section{СПИСОК ЦИТИРОВАННОЙ ЛИТЕРАТУРЫ}

1. Марченко В. А. Операторы Штурма-Лиувилля и их приложения. Киев: Наукова думка, 1977. 2. Келдыш М. В. // Докл. АН СССР. 1951. Т. 77. №1. С. 11-14. 3. Шкаликов А. А. // Функцион. анализ и его прилож. 1976. Т. 10. № 4. С. 69-80. 4. Маламуд М. М., Оридорога Л. Л. // Функцион. анализ и его прилож. 2000. Т. 34. № 3. С. 88-90. 5. Тарапова Е. И. // Теория функций, функцион. анализ и их прилож. 1979. Т. 31. С. 157-160. 6. Тарапова Е. И. // Теория функций, функцион. анализ и их прилож. 1979. Т. 33. С. 82-87. 7. Oridoroga L. L. // Доповіді НАН України. 2001. № 3. P. 34-39. 8. Oridoroga L. L. // Methods of Functional Analysis and Topology. 2001. V. 7. № 1. Р. 82-87. 9. Маламуд M. M. // Тр. MMO. 1999. Т. 60. С. 199-258. 